\title{
Single-shot Mesoporous Silica Rods Scaffold for Induction of Humoral Responses Against Small Antigens
}

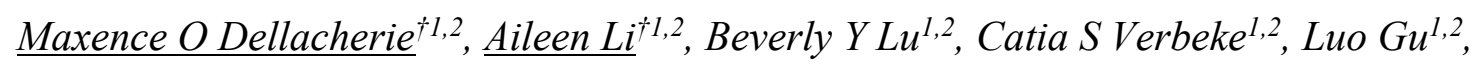

Alexander G. Stafford ${ }^{2}$, Edward J. Doherty ${ }^{1,2}$ and David J. Mooney ${ }^{1,2 *}$

$\digamma$ These authors contributed equally to this work

*Correspondence to: mooneyd@seas.harvard.edu

1. John A. Paulson School of Engineering and Applied Sciences, Harvard University, Cambridge, MA, USA

2. Wyss Institute for Biologically Inspired Engineering, Harvard University, Boston, MA, USA

Keywords: antibody, vaccine, single-shot, mesoporous silica, scaffold, dendritic cell, peptide 


\begin{abstract}
Vaccines have shown significant promise in eliciting protective and therapeutic responses.
\end{abstract}

However, most effective vaccines require several booster shots, and it is challenging to generate

potent responses against small molecules and synthetic peptide antigens often used to increase

target specificity and improve vaccine stability. As continuous antigen uptake and processing by

APCs and persistent toll-like receptor (TLR) priming have been shown to amplify antigen specific

humoral immunity, we explored whether a single injection of a mesoporous silica micro-rod

(MSR) vaccine containing synthetic molecules and peptides can effectively generate potent and

durable antigen-specific humoral immunity. A single injection of the MSR vaccine against a

gonadotropin-releasing hormone $(\mathrm{GnRH})$ decapeptide elicited highly potent anti-GnRH response

that lasted for over 12 months. The MSR vaccine generated higher titers than bolus or alhydrogel

alum vaccine formulations. Moreover, a MSR vaccine directed against a Her2/neu peptide within

the Trastuzumab binding domain showed immunoreactivity to native Her2 protein on tumor cell

surface and, when directed against nicotine, generated long-term anti-nicotine antibodies.

Mechanistically, we found that the MSR vaccine induced persistent germinal center (GC) B-cell

activity for more than 3 weeks after a single injection, generation of memory B cells, and that at

least 7 days of immunostimulation by the vaccine was required to generate an effective humoral

response. Together, these data suggest that the MSR vaccine represents a promising technology 

available under aCC-BY-NC-ND 4.0 International license.

for synthetic antigen vaccines to bypass the need for multiple immunizations and enhance longterm production of antibodies against endogenous antigens in the context of reproductive biology, cancer, and chronic addiction. 


\section{Introduction}

Immunotherapies are rapidly becoming a standard approach for cancer and chronic infectious disease treatment ${ }^{[1]}$. Vaccines can induce strong protective and therapeutic responses against many forms of live and attenuated virus, toxoids, irradiated autologous tumor cells and protein subunits. However, most effective vaccines require several boosts after the primary immunization ${ }^{[2]}$. These dosing regimens are costly, increase hospital/clinic visitation for patients and are especially challenging in regions where limited healthcare access poses a major logistical barrier to disease treatment and management ${ }^{[2,3]}$. An effective single-shot vaccine could overcome these limitations. Most approaches for single-shot vaccines in clinical testing to date have used depots of antigens that were released in a controlled manner for months after immunization ${ }^{[2,4-6]}$. However, passive diffusion of the antigen and adjuvant to the lymph nodes alone is likely unable to maximally and rapidly stimulate immune cells and provide life-long benefit. Indeed, besides Bcell stimulation by antigen, humoral responses development requires a complex immune cascade involving antigen-presenting cells (APC) activation, subsequent T-helper cell generation and finally cognate T-cell/B-cell interactions. However, these steps that occur in parallel during infection are not easily recapitulated by current vaccine technologies. Therefore, achieving a 
robust and lasting immune response after a single injection of a vaccine remains an important

challenge.

Small molecules and peptides have many potential advantages as antigens but generating

potent responses is challenging. The use of small antigens in vaccine can help focus the immune response with high epitope specificity and biological activity while avoiding off-target effects. For instance, a synthetic peptide vaccine against subtypes of influenza using Keyhole limpet hemocyanin $(\mathrm{KLH})$ as a carrier protein generated anti-hemagglutinin antibodies and neutralizing activity ${ }^{[7]}$. Peptides can be particularly advantageous for vaccine development as they can be chemically synthesized quickly and with low variability. Their production can be easily up-scaled and they have good safety and stability ${ }^{[8]}$. In contrast to the time and cost-intensive approaches that require the production of recombinant protein, attenuated virus or cancer cell culture, synthetic peptides can expedite the manufacturing of personalized cancer vaccines and ensure the timely development of vaccines against new pandemics ${ }^{[9-11]}$. Peptides are also readily presented by antigen presenting cells (APCs) to induce subsequent effector $\mathrm{T}$ cell and humoral responses. However, synthetic peptide vaccines generally cannot confer long-lived, therapeutic benefit. This limitation is likely caused by a combination of 1) short peptide half-life due to rapid systemic clearance, 2) weak immunogenicity due to lack of a co-stimulatory signal and either absent or 
suboptimal T-helper epitopes co-presentation, and 3) absence of B-cell receptor (BCR) crosslinking due to lack of multivalency ${ }^{[8,12,13]}$.

An example of an attractive target for vaccination is gonadotropin-releasing hormone $(\mathrm{GnRH})$, a decapeptide hypothalamic hormone that controls male spermatogenesis, and female estrogen and follicular development ${ }^{[14]}$. GnRH determines both fertility and sexual behavior and is highly conserved among mammals. GnRH activity has also been implicated in the progression of several types of malignancies including prostate, breast and ovarian cancers ${ }^{[15-17]}$. Blocking GnRH activity using androgen deprivation therapies (ADTs) and gene therapy have shown preclinical promise, but such therapies have strong toxic side effects and lack long term potency ${ }^{[12,16]}$. Active immunizations against GnRH using various carrier proteins such as KLH, ovalbumin (OVA) and diphtheria toxoid (DT) have been shown to decrease sexual organ function, reduce testosterone levels in patients with advanced prostate cancer and reduce reproductive capability in feral animals as a humane alternative to surgical desexing ${ }^{[16-18,20]}$. However, these vaccines require multiple immunizations and the effects are variable and not potent enough to produce long-term immune-castration ${ }^{[21]}$.

Here we propose a single injection vaccine platform using a mesoporous silica micro-rod (MSR) vaccine system to generate high antibody titers against synthetic peptide and other small antigens. Biomaterials have shown substantial potential to integrate with conventional vaccine 
approaches by, for example, extending the half-life of immune-modulating drugs and controlling the presentation of adjuvants and antigens ${ }^{[22-24]}$. In particular, biomaterial scaffold vaccines have been demonstrated to locally recruit and program host antigen presenting cells (APCs) to induce effective innate and adaptive immunity ${ }^{[25,26]}$. Using this approach, an implantable poly(lactideco-glycolide) (PLGA) based scaffold cancer vaccine was shown to induce complete melanoma regression in subsets of mice ${ }^{[27]}$. To eliminate the need for surgical implantation, we recently developed a new approach of injectable scaffolds using mesoporous silica rods (MSR) microparticles ${ }^{[28,29]}$. We demonstrated that MSR microparticles could spontaneously assemble into a 3D scaffold after subcutaneous injection; the macropores formed by random particle stacking allowed for cell infiltration and active cell-scaffold interactions. The controlled release of GM-CSF and CpG from the MSR vaccine modulated host APC infiltration into the vaccine and, consequently, continuously programmed host APCs. Continuous antigen uptake and processing by APCs and persistent toll-like receptor (TLR) priming have been shown to amplify antigen specific humoral immunity. Overall, we reasoned that concomitant release of antigen from MSR scaffold and local APC activation could enable continuous B-cell activation in the lymph node and enhance cognate T-helper cell priming (Figure 1). Therefore, we hypothesized that a single injection of the MSR vaccine could generate robust and long-lasting humoral immunity and enhance the response compared to traditional vaccine approaches. We focus on GnRH as a model 
antigen, but also explore this concept with a small molecule epitope nicotine and a Her2 peptide epitope within the Trastuzumab-binding domain.

\section{Results}

\subsection{Single injection of MSR vaccine with GnRH peptide conjugated to OVA (GnRH-OVA)}

\section{induced higher and more durable anti-GnRH humoral response compared to bolus vaccines.}

To evaluate whether a CD4 helper T cell epitope was necessary to generate an anti-GnRH

response, we incorporated either free GnRH peptide or GnRH peptide conjugated to the carrier

protein ovalbumin (OVA) into the MSR vaccine. MSR vaccines were formulated to contain $1 \mu \mathrm{g}$

GM-CSF and $100 \mu \mathrm{g}$ CpG-ODN, as previously described, and either $100 \mu \mathrm{g}$ free GnRH or $100 \mu \mathrm{g}$

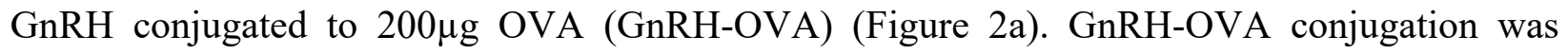

confirmed using mass spectrometry (Figure S1). The loading efficiencies of free GnRH and

GnRH-OVA onto the MSRs were $91 \pm 2 \%$ and $85 \pm 1 \%$, respectively (Figure S2). Vaccines were

injected into the subcutaneous tissue of mice, and antibody titers were subsequently measured

using ELISA. Strikingly, a single immunization with the MSR vaccine containing GnRH-OVA

elicited strong titers of anti-GnRH IgG1 (Figure 2b) and IgG2a (Figure 2c) serum antibody for

over 12 months. In comparison, immunization with MSRs loaded with unconjugated GnRH did

not elicit detectable anti-GnRH titer. To confirm the serum anti-GnRH antibody recognized the 
GnRH peptide sequence specifically, serum from animals vaccinated with the MSR GnRH-OVA vaccine were tested using an ELISA coated with GnRH peptide or a scrambled peptide (CRSYGPLHEWG) (Figure S3a). The serum antibody recognized the GnRH peptide in a dilutiondependent manner but did not recognize the scrambled peptide. Finally, to confirm that the antiGnRH antibody response was specific to the MSR GnRH-OVA vaccine, we immunized mice with

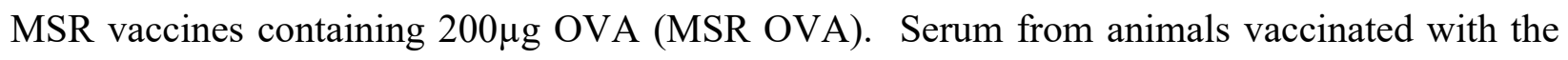
MSR OVA vaccine did not elicit any detectable anti-GnRH IgG1 (Figure S3b) or IgG2a (Figure S3c) titers.

We next investigated whether the MSR vaccine can elicit higher antibody titers compared to traditional bolus immunizations (Figure 2d). Mice were again immunized with a single injection of the MSR vaccine loaded with $1 \mu \mathrm{g}$ GM-CSF, $100 \mu \mathrm{g}$ CpG-ODN and 100 $\mu \mathrm{g}$ GnRH conjugated to $200 \mu \mathrm{g}$ OVA or a single injection of a bolus vaccine containing $1 \mu \mathrm{g}$ GM-CSF, $100 \mu \mathrm{g}$ CpG-ODN and $100 \mu \mathrm{g}$ GnRH conjugated to $200 \mu \mathrm{g}$ OVA in PBS (GnRH-OVA Bolus Vax) (Figure 2d). The MSR vaccine induced significantly higher anti-GnRH IgG1 (Figure 2e) and IgG2a (Figure 2f) antibody titers compared to the bolus vaccine. The effect was also durable, lasting for at least 100 days post immunization. Impressively, the MSR vaccine induced rapid onset of antibody production. On day 14, the titer elicited by the MSR vaccine was 35 fold and 80 fold higher than that elicited by the bolus vaccine for IgG2a and IgG1 subtypes, respectively. Keyhole Limpet 
Hemocyanin (KLH) is the most commonly used carrier protein immunogen to induce antibody

production, as it is highly immunogenic due to its large molecular weight and repetitive structure,

which allow for high valency antigen conjugates ${ }^{[30]}$. Using GnRH-KLH conjugate as the antigen,

MSR vaccine again significantly enhanced the total serum anti-GnRH IgG response compared to a bolus KLH-GnRH vaccine (Figure 2g). At the peak of the response, the titer elicited by the MSR vaccine was nearly 10 fold higher than that elicited by the bolus vaccine.

\subsection{Single injection of MSR vaccine induced persistent Germinal Center (GC) activity.}

A strong humoral response depends on activated B cells forming GCs in secondary lymphoid organs, where they further undergo somatic hypermutation and isotype switching, and eventually differentiate into plasma cells that can rapidly produce antibodies upon secondary encounter with the antigen ${ }^{[31]}$. Therefore, we investigated whether a single injection of the MSR GnRH-OVA vaccine can stimulate enhanced GC formation compared to the conventional bolus vaccination. The MSR GnRH-OVA vaccine induced a prolonged immune response, as indicated by the increased total cell number in the dLN from 7 days to 25 days after immunization (Figure 3a). In contrast, although the bolus GnRH-OVA vaccine enhanced dLN cell number on day 7, the response rapidly dissipated. Next, the percentage and total cell number of GC B cells in the dLN were quantified using the markers $\mathrm{B} 220^{+} \mathrm{GL}^{+}$(Figure $3 \mathrm{~b}$ and $3 \mathrm{c}$ ) and $\mathrm{B} 220^{+} \mathrm{GL}^{+} \mathrm{PNA}^{+}$(Figure 
S4a and S4b, primary gating plots shown in Figure S5) using flow cytometry. By day 7 after immunization, both the MSR vaccine and the bolus vaccine led to an enhanced GC response.

Notably, the enhanced GC response in the MSR vaccinated animals persisted until after day 25.

The number of GC B cells in the MSR vaccinated mice was approximately 10 fold higher than those in the bolus vaccine treated mice on days 14 and 25 . In contrast, the GC response in the bolus vaccinated animals sharply dissipated after 7 days. These findings were further supported by immunofluorescence staining of GC B cells in the dLNs over time (Figure 3d).

\subsection{Single injection of MSR vaccine improves memory B-cell generation.}

Efficient prophylactic vaccination requires the induction of long-term immunological memory to protect against pathogen encounter at a later time. Memory B-cells generated by vaccination can persist for years and be reactivated upon antigen encounter to produce rapid antibody responses ${ }^{[31]}$. Therefore, we compared the capacity of bolus and MSR-based vaccines to induce such responses. To detect the rare memory B-cells generated by the vaccine, we used fluorescent protein phycoerythrin (PE) as both the antigen and the detection reagent (Figure 4a)

[32]. 10-12 weeks after immunization, several weeks after all vaccine components had cleared

(Figure 4b), cells from the draining lymph node (dLN), spleen and bone marrow were stained with PE, enriched for PE-binding cells using anti-PE magnetic and finally, PE-specific B-cells were 
detected by flow cytometry (Figure S6). Compared to naïve mice, MSR-immunized mice showed greatly increased numbers of $\mathrm{PE}^{+} \mathrm{B} 220^{+} \mathrm{B}$-cells in the draining lymph node and spleen. By contrast, bolus immunization did not provide a significant boost in antigen-specific B-cells compared to the naïve baseline. No differences were observed in the bone marrow for any of the groups (Figure 4c). Overall, this data illustrates that MSR vaccine can enhance memory B-cell generation compared to traditional bolus vaccination.

\subsection{MSR scaffold persistence and cell-recruitment is required for robust humoral response}

\section{generation}

To begin to investigate how the dynamics of immune cell recruitment and antigen availability impacted on the humoral response generated by MSR scaffolds, we determined the kinetics of antigen presentation and cellular infiltration in GnRH-OVA MSR vaccines. Following subcutaneous injection, the vaccine formed a dynamic nodule which peaked in size on day 7 and slowly degraded over the next several weeks. Scaffolds were undetectable 45 days after injection (Figure 5a). MSR scaffolds released antigen slowly with $27 \pm 3 \%$ of the GnRH-OVA cargo desorbing in the first 12 hours followed by minimal release ( $10 \pm 1 \%$ of total load) over 25 days in vitro (Figure $5 \mathrm{~b}$ ). To track antigen in scaffolds in vivo, we immunized mice with MSR vaccines formulated with fluorescent-OVA as the antigen (MSR-OVA*) and imaged the vaccine site 7, 14 
and 30 days after injection using confocal microscopy. OVA* fluorescence was detected at all timepoints, with scaffold area decreasing after day 7, confirming scaffold dynamics and the persistence of antigen for up to 30 days (Figure 5c).

Next, the kinetics of cell recruitment by the MSR GnRH-OVA vaccines was quantified. The MSR vaccine has been engineered to recruit and activate host immune cells by incorporating and releasing GM-CSF and CpG-ODN ${ }^{[28]}$. Consistent with our previous work, host immune cells were shown to infiltrate the MSR scaffold in a time dependent manner, peaking at day 7 (Figure 5d). $\mathrm{CD} 11 \mathrm{c}^{+} \mathrm{CD} 11 \mathrm{~b}^{+} \mathrm{DCs}$ were detectable at day 1 post immunization, their percentage peaked at day 3 and dropped sharply at day 5, followed by a secondary increase from day 7 to day 14 (Figure 5e). Recruited DCs were activated by the released $\mathrm{CpG}-\mathrm{ODN}$, as indicated by a higher percentage of $\mathrm{CD} 11 \mathrm{c}^{+} \mathrm{CD}^{+} 6^{+} \mathrm{DCs}$ on day 3 after immunization (Figure $5 \mathrm{f}$ ). The percentage of activated DCs dropped from day 3 to day 5, likely due to their homing to the draining lymph node (dLN). The percentage of activated DCs in the scaffold remained low from day 5 to day 14. Together, these data suggest the MSR GnRH-OVA vaccine induces rapid DC infiltration, activation and exfiltration, and serves as a site of sustained antigen presentation that may program immune cells for extended periods.

To investigate the relationship between the infiltrated immune cells and the anti-GnRH titer, the MSR vaccines were explanted at days 1, 3, 5, 7 and 15 after immunization (Figure 6a). 
Vaccination durations of less than 5 days resulted in no significant overall anti-GnRH IgG1 (Figure

6b) or IgG2a (Figure 6c) titers. Vaccines that were explanted on day 5 led to detectable amounts

of IgG1 titer on day 14 and 20, but the response was transient and no titers were detected from this

condition after 20 days. In contrast, a vaccination duration of 7 days or greater led to prolonged

anti-GnRH IgG1 (Figure 6d) and IgG2a (Figure 6e) titers. Vaccines that were explanted on day 7

resulted in a significantly slower onset of titer production compared to vaccines that were

explanted on day 15 and those that were not explanted. There was no difference in titer levels

between vaccines that were explanted on day 15 and those that were not explanted. Together, this

data suggest that vaccine duration of 15 days is necessary to generate both rapid onset and durable

high anti-GnRH titers.

To further probe the importance of cell recruitment for antibody generation, we created

MSR vaccines with diminished cell recruitment capabilities. We previously reported that the compression of MSR scaffolds into a tablet (Monolith) decreased cell infiltration by reducing the 3D space available for immune cell infiltration ${ }^{[28]}$. In addition, we encapsulated MSR solution into a nanoporous alginate hydrogel (MSR-Alginate) to prevent cell ingress (Figure 7a). When the 
modified GnRH-OVA MSR vaccines were used, they generated 1 to 2 orders of magnitude lower anti-GnRH titer than pristine MSR (Figure 7b).

\subsection{Broad potential of MSR for vaccination against small molecules and linear peptide}

antigens.

To demonstrate the broader potential of MSR vaccines, we tested their capacity to generate antibodies against different small molecule and linear peptide antigens (Figure 8a). Besides protein-derived antigens, there is broad interest in eliciting neutralizing antibodies against small molecules such as toxins or psychoactive substances to nullify their action ${ }^{[33,34]}$. For example, vaccination has been investigated to produce antibodies that bind nicotine in the bloodstream to reduce the rewarding effect of tobacco and assist in smoking cessation ${ }^{[33,35]}$. A single injection of MSR vaccines containing OVA-conjugated nicotine could induce high titers of anti-nicotine IgG, which remained elevated 6 months post injection (Figure 8b).

Vaccines that generate antibody responses using carrier proteins may require cumbersome recombinant protein production and chemical conjugation processes, which can be costly, lead to batch-to-batch variability, and elicit off-target antibody responses against other $\mathrm{T}$ and $\mathrm{B}$ cell epitopes on the carrier protein itself ${ }^{[36]}$. To circumvent this issue, we designed peptide constructs that incorporate a promiscuous T-helper cell epitope derived from measles virus fusion protein 
(MVF), a flexible linker, and the B-cell epitope of interest. In a first example, we targeted the oncogenic protein HER2/neu, a surface receptor that has been successfully targeted by monoclonal antibodies for the treatment of Her2 ${ }^{+}$breast and gastric cancer ${ }^{[37]}$. To specifically induce therapeutic antibodies, we used Her2 ${ }_{613-626}$ as the B-cell epitope, as it is within the portion of the protein bound by trastuzumab (Herceptin), a clinically approved monoclonal antibody that antagonizes the function of the Her2 receptor. Compared to a bolus, the MSR vaccine significantly enhanced both the IgG1 and IgG2a titer generated against the Her2 protein (Figure 8b). Moreover, serum IgG from MSR mice could bind to the Her2 ${ }^{+}$SK-BR-3 breast cancer cell line but not to CT26 Her2- cells. In contrast, serum from a bolus vaccine showed no detectable binding to Her2expressing cancer cells (Figure 8d-e).

Finally, we investigated the anti-GnRH response generated against a synthetic MVFGnRH peptide antigen, comparing our MSR technology with a bolus vaccine and MVFGnRH formulated in the gold standard adjuvant Alum. In both cases, the total IgG titer induced by the MSR was on average an order of magnitude higher following a single injection (Figure $8 \mathrm{f}$ and $\mathrm{g}$ ). Overall, this 
data illustrates the potential of MSR as a vaccine platform to elicit robust antibody responses against small antigens with otherwise low immunogenicity.

\section{Discussion}

The findings of this study demonstrate that a single injection of the MSR vaccine elicits potent and durable serum antibody titers against small antigens, including small molecules and short peptides. Both peptide-carrier protein constructs (e.g. GnRH-OVA and GnRH-KLH) and tandem peptide constructs (e.g. MVFGnRH and MVFHer2) demonstrated that the MSR vaccine induced more potent and durable humoral responses than traditional vaccinations. Notably, the MSR GnRH-OVA vaccine generated high anti-GnRH titers even 12 months after vaccination. Although carrier proteins can significantly increase immune responses against a peptide, this approach has several limitations. First, chemical coupling of specific residues on the carrier protein and the peptide can be difficult to control, and may lead to variability in vaccine response ${ }^{[38]}$. Second, because the carrier protein contains multiple antigenic epitopes, there is likely skewed clonal dominance of carrier-specific B cell responses ${ }^{[36,39]}$. For these reasons, tandem peptide constructs containing a single CD4 T-helper epitope and the target peptide were also explored.

The MSR MVFGnRH vaccine produced 10-20 fold higher antibody titers compared to the bolus formulations, and MSR vaccination led to faster antibody production and overall higher response 
compared to a conventional Alum adjuvanted vaccine. Incorporating the antigen into the MSR scaffold likely resulted in multivalent display of the antigen on the MSR pores and surface, which may facilitate BCR crosslinking and enhance B cell activation for stronger humoral responses.

The MSR vaccine generated antibodies against a portion of the Her2 epitope that exhibited immunoreactivity to the native Her2 structure on a tumor cell surface. While Trastuzumab can inhibit tumor growth, mAb therapy lacks long-term efficacy due to the limited half-life time of the immunoglobulins, and repeated administration of the mAb results in many side effects ${ }^{[40-42]}$. It has been demonstrated that Trastuzumab binds to the portion of the Her2 extracellular domain spanning between residues 563-626 that contains three loops ${ }^{[43]}$. Previous studies identified a number of peptides that mimic portions of the Her2 domain at the Her2/Trastuzumab interface ${ }^{[44-}$ 46]. Repeated vaccination using these peptides led to antibodies that could recognize Her2/neu displayed on a cell surface and induced Her2 internalization in a similar manner as Trastuzumab. Here we chose a short and linear peptide Her2613-626 to evaluate the robustness of the MSR vaccine. Other studies using the same peptide construct showed that multiple vaccinations were required to raise high titers ${ }^{[46]}$. In comparison, a single injection of the MSR vaccine was able to induce antibody titers up to 2 orders of magnitude higher than the bolus vaccine. In particular, the antibodies recognized Her2 displayed on human breast tumor cells, whereas the antibodies produced by the bolus vaccine did not show any significant binding. Although linear peptides are 
highly flexible and inexpensive to synthesize, they can adopt a variety of conformations in buffer and only a subset of these conformations are ultimately responsible for antibody reactivity. Therefore, future studies should explore various cyclic peptides mimicking the Her2 loop to confer antibody specificity, and evaluate the anti-tumor efficacy. Overall, these data suggest that the MSR vaccine can likely promote continuous in situ antibody production, generate focused and robust humoral anti-tumor immunity and form long-term immunological memory.

The relationship between MSR vaccine duration and the kinetics and maintenance of the humoral response suggests the importance of prolonged DC programming in the MSR vaccine. The DC profile in the MSR vaccine showed two distinct waves; the first one peaked on day 3 and rapidly diminished by day 5 , and the second initiated after day 7 . The first wave of DCs was likely due to recruitment mediated by release of GM-CSF. The residence of these DCs in the vaccine was transient, likely due to activation by the released CpG-ODN and homing to the dLN. The second wave of DCs was possibly a result of cytokines and chemokines secreted by the first wave of DCs and other immune cells. Interestingly, the first wave of DCs exhibited an activated phenotype, while the DCs in the second wave were mostly immature. Explanting the MSR vaccine between day 1 and day 5, before the first wave of DCs could be accumulated and subsequently home to the dLNs, resulted in no significant humoral response. When the vaccine was explanted after day 7, the vaccine effectively generated and maintained potent humoral responses over 100 
days. Studies have shown that prolonged durations of vaccination ( $>16$ days) using engineered

biomaterial scaffold vaccines significantly augmented persistent CTL responses locally and

slowed tumor progression ${ }^{[47]}$. Here, we showed that vaccine duration was also important in

humoral responses. Explanting the scaffolds not only impacts local APC activation by MSR but

also suppresses antigen delivery to draining lymph nodes. However, when the capacity of the MSR

scaffold for cell infiltration was reduced (using MSR monolith or alginate encapsulation) and

antigen presence maintained, the antibody was also partially diminished. This data suggest that the

high antibody titer generated by MSR are, in part, generated by APC recruitment and trafficking

through the scaffolds.

The enhanced humoral responses generated by the MSR vaccine correlated strongly with

persistent GC activity. After encountering an antigen, activated B cells form GCs where they

switch their immunoglobulin constant region from $\operatorname{IgM}$ to $\operatorname{IgG}, \operatorname{IgA}$ or $\operatorname{IgE}$, and undergo somatic

hypermutation in the variable region to produce antibodies with high specificity and avidity ${ }^{[32]}$.

A number of biomaterial-based vaccine systems have been shown to stimulate persistent GC activity. For instance, poly(lactic-co-glycolic acid) (PLGA) nanoparticles containing multiple

TLR ligands induced active GCs for $\sim 45$ days, and multilamellar lipid vesicle based LN-targeting

nanoparticles showed superior GC formation compared to bolus formulations on day $14^{[48,49] .}$

However, these strategies required multiple injections to achieve this effect. In contrast, only a 
single injection of the MSR vaccine induced persistent GC activity for over 30 days. Specialized

B cells that emerge from the GC secrete high affinity antibodies and can also differentiate into

long-lived plasma and memory cells, which are necessary to generate life-long antibody

production and rapid immune re-activation upon antigen re-encounter, respectively ${ }^{[31]}$. Consistent

with the sustained GC responses, MSR vaccines generated long-term antibody production and

higher numbers of memory B-cells than their bolus counterparts. Overall, this data illustrates to

the potential of the MSR vaccines to generate both therapeutic and prophylactic antibody

responses. The duration of the GC activity after immunization with the MSR vaccine also

correlated with the amount of time the MSR vaccine remained visible at the injection site. Future

studies could explore how varying MSR vaccine degradation kinetics impacts GC activity and

antibody production. In addition, the kinetics of antigen presentation after vaccination with the

MSR system could likely be further optimized, as recent studies have demonstrated a dramatic

impact of the antigen presentation profile on the immune response ${ }^{[50,51]}$. The role of persistent

antigen presentation by different subtypes of APCs is also an important topic for further study.

\section{Conclusion}

The findings of these studies suggest the MSR vaccine may have high utility as a singleinjection platform to generate potent humoral responses. We demonstrated that MSR vaccines 
induce high and persistent antibody titers against small challenging antigens with relevance to a variety of therapeutic applications including addiction therapy, immune-castration for feral animal population control and cancer treatment. Different subunits antigens were and can easily be swapped using the MSR system, which could therefore have significant impact in other areas, such as chronic bacteria or viral infection, and neurodegenerative diseases.

Compared to conventional vaccination strategies, MSR scaffolds improved the magnitude of antibody response, boosted GC persistence, and increased memory B-cell generation, all desirable features of efficient immunization schemes. The magnitude of the humoral response generated was dependent on scaffold persistence and local cell recruitment, which could be further modulated to refine MSR vaccines. These findings have broader implications for our understanding of current vaccines and the design of new vaccine technologies.

\section{Experimental Section}

Peptides and proteins. All peptides used in this study were synthesized at least $95 \%$ purity from

Peptide 2.0. Peptide sequences are as follows: CGnRH (CEHWSYGLRPG), scrambled CGnRH

peptide (CRSYGPLHEWG), MVFGnRH (KLLSLIKGVIVHRLEGVEGPSLEHWSYGLRPG),

MVFHer2 (KLLSLIKGVIVHRLEGVEGPSLIWKFPDEEGACQPL). Endotoxin free ovalbumin 
was purchased from Invivogen (vac-pova), and maleimide activated KLH was purchased from

ThermoFisher Scientific (77605).

MSR synthesis and MSR vaccine formulation. MSRs were fabricated as described previously.

Briefly, 4g of P123 surfactant (average Mn 5800, Sigma-Aldrich) were dissolved in $150 \mathrm{~g}$ of

1.6M HCl solution and stirred with $8.6 \mathrm{~g}$ of tetraethyl orthosilicate (TEOS, 98\%, Sigma-Aldrich)

at $40{ }^{\circ} \mathrm{C}$ for $20 \mathrm{~h}$, followed by aging at $100{ }^{\circ} \mathrm{C}$ for 24 hours. TEOS was extracted in $1 \% \mathrm{EtOH}$ in

$\mathrm{HCl}$ at $80{ }^{\circ} \mathrm{C}$ for 18 hours. To prepare the MSR vaccines, $4 \mathrm{mg}$ of the MSR were adsorbed with

$100 \mu \mathrm{g}$ murine class B CpG-ODN (sequence TCCATGACGTTCCTGACGTT, IDT) and varying

amounts of antigen (peptide or peptide-carrier protein conjugate) for 8 hours at room temperature

under shaking, and subsequently lyophilized. Separately, $1 \mathrm{mg}$ of the MSRs were loaded with

murine GM-CSF (Peprotech) for 1 hour at $37^{\circ} \mathrm{C}$ under shaking. The MSRs were combined and

resuspended in cold PBS prior to immunization. To determine loading efficiency, MSR loaded

with antigen were resuspended in PBS, centrifuged to pellet MSR and the supernatant was 
collected. Antigen concentration was determined by measuring the concentration of remaining antigen in supernatant using a BCA protein quantification kit (Pierce)

Peptide protein conjugation. To conjugate CGnRH to ovalbumin (OVA), OVA was first reacted with 30 molar excess of sulfo-SMCC (ThermoFisher Scientific) in PBS for 1 hour at room temperature. Subsequently, the maleimide-OVA was desalted to remove the excess sulfo-SMCC. Separately, CGnRH or CHer2 was reduced using 2 molar excess of TCEP (ThermoFisher Scientific) for 1 hour at room temperature. $100 \mu \mathrm{g}$ of Maleimide-OVA was then reacted with $100 \mu \mathrm{g}$ the CGnRH peptide in PBS for 12 hours at room temperature under shaking.

Nicotine protein conjugation. Ovalbumin (OVA) was reacted with succinic anhydride (Sigma) for $2 \mathrm{~h}$ in $0.1 \mathrm{M}$ borate buffer at a 1:50 molar ratio (1:2 amine to succinic anhydride). The resulting product was buffer-exchanged to MES buffer, pH 5.8 and reacted with 600 molar excess of 1Ethyl-3-(3-dimethylaminopropyl)carbodiimide (EDC, Sigma) and 200 molar excess racemic aminomethylnicotine for $20 \mathrm{~h}$. Finally, the OVA-Nicotine product was buffer-exchanged to PBS 
for use in vaccination studies. Bovine Serum Albumin (BSA)-Nicotine conjugates were produced using the same procedure to detect anti-nicotine serum antibody by ELISA.

Immunizations. Unless otherwise noted, all in vivo studies were carried out using female C57BL/6J mice (Jackson Laboratories) between 6 to 10 weeks old at the beginning of the experiment. MSR vaccines were resuspended in 150ul of cold PBS and injected, via an $18 \mathrm{G}$ needle, subcutaneously in the intrascapular region with the mouse under brief isoflurane anesthesia. We have demonstrated that the MSR vaccine can be injected using 23G needles, but we used $18 \mathrm{G}$ needles in these studies to be consistent with our previous studies. All animal studies were performed in accordance with NIH guidelines, under approval of Harvard University's Institutional Animal Care and Use Committee.

Sera titer analysis using ELISA. Peripheral blood was collected periodically after immunization.

Sera samples were analyzed for IgG1 (BD Biosciences), IgG2a (BD Biosciences) or total IgG

(Biolegend) levels using ELISA. Briefly, ELISA plates were coated overnight in $4{ }^{\circ} \mathrm{C}$ with $30 \mathrm{ug} / \mathrm{ml}$ of $\mathrm{GnRH}, 10 \mathrm{ug} / \mathrm{ml}$ Her2 or $5 \mathrm{ng} / \mathrm{mL}$ BSA-Nicotine in PBS. ELISA was performed 
according to established procedures, and anti-GnRH or anti-Her2 titers were defined as the lowest serum dilution at which the ELISA OD reading was equal to OD value 0.2.

Germinal center characterization. To analyze GC formation, dLNs were isolated on days 7, 14, 25 and 50 after immunization. Cells were enumerated, and stained with anti-mouse B220 (eBioscience), anti-mouse GL7 (eBioscience) and Rhodamine-PNA (Vector) for 15 minutes on ice. Cells were washed and assessed using flow cytometry (BD Fortessa or BD LSR-II).

Lymph node histology. dLNs were fixed for 1 hour with $4 \%$ paraformaldehyde (Thermofisher Scientific), embedded in Tissue-tek OCT (VWR) and cryo-sectioned using a Leica CM1950 Cryostat. Various sections from one LN were stained with anti-B220 (eBioscience) and anti-GL7 (eBioscience) and visualized using confocal (Zeiss LSM 710).

Cell isolation from MSR scaffolds explanted from animals. Scaffolds were excised on day 1, 3, 5, 7 or 15 after immunization. The tissues were processed through mechanical disruption and suspended in PBS. The resulting cell suspension was then filtered through a $40 \mu \mathrm{m}$ cell strainer to isolate the cells from the larger sized MSRs. The cells and small remaining MSR particles were pelleted, washed with cold PBS, and counted (Beckman-Coulter). The portion of cells in the 
mixture of cells and small silica particles was accessed in SSC and FSC gating in flow cytometry

(BD LSRII or BD Fortessa). Based on the counts from Coulter counter and the percentage of cells determined from FACS gating, the number of live cells in the MSR scaffolds could be calculated.

Analysis of DC recruitment to MSR scaffolds. APC-conjugated CD11c (eBioscience), FITCconjugated CD11b (eBioscience) stains were conducted for DC and leukocyte recruitment analysis, and APC-conjugated CD11c and PE-conjugated CD86 stains were conducted for DC maturation analysis. Cells were stained with 7-AAD and fluorophore conjugated antibody for 15 minutes on ice, washed thoroughly and analyzed using flow cytometry (BD LSRII or BD Fortessa). Cells were first gated according to the viability channel, live cells were then gated according to positive FITC, APC and PE using isotype controls, and the percentages of cells staining positive for each surface antigen were recorded.

Analysis of memory B-cells. Mice were immunized as described above. In brief, each mouse received a subcutaneous injection of $1 \mu \mathrm{g}$ GM-CSF, $100 \mu \mathrm{g}$ CpG and $30 \mu \mathrm{g}$ PE (R-PE, Thermosfisher) in PBS (Bolus) or loaded on MSR (MSR) as described above. 10-12 weeks later, mice were euthanized and their draining lymph nodes, spleen and a lower limb harvested. Lymph nodes and spleens were processed in to single cells suspensions as described previously ${ }^{[52]}$. 
Marrow was flushed off cleaned bones using a $21 \mathrm{G}$ needle and further processed into a single cell suspension. As described previously $\left[{ }^{32}\right]$, cells were stained with $1 \mu \mathrm{g} \mathrm{PE}$, enriched for $\mathrm{PE}^{+}$cells using anti-PE magnetic beads (Miltenyi Biotec). Cells were then enumerated and stained with Pacific Blue anti-mouse CD4, CD8, F4/80, Gr-1, Cd11c, FITC anti-mouse B220 (eBioscience) and Near-IR Fixable LIVE/DEAD dye (Thermofisher). Cells were washed and analyzed by flow cytometry (BD LSR-II) to detect CD4-CD8-F4/80-Gr1-CD11 c-B220+ ${ }^{-} E^{+}$memory B-cells.

Mass spectrometry. GnRH-OVA conjugate was synthesized as described above and desalted. OVA and the conjugates were analyzed using Agilent 6460 Triple Quadrupole Mass Spectrometer equipped with Agilent 1290 uHPLC.

Preparation of modified MSR vaccine for reduced cell recruitment. MSR vaccines were formulated as described above with the following modifications. $200 \mu \mathrm{g}$ OVA conjugated to $100 \mu \mathrm{g}$ GnRH peptide (OVA-GnRH) was loaded onto per 4mg MSR, flash frozen in liquid nitrogen and lyophilized. $1 \mu \mathrm{g}$ GM-CSF was loaded per 1mg MSR. MSR monolith were prepare by mixing the GnRH-OVA/MSR lyophilized particles and the MSR/GM-CSF solution and compressing them into an $8 \mathrm{~mm}$ table using a lab press. Gel-embedded vaccines (Alginate-MSR) were made using norbornene-modified alginate $(\mathrm{Nb}: \mathrm{Alg})$ and tetrazine-modified alginate (Tz:Alg) that were 
prepared as described previously ${ }^{[53]}$. 1.12mg Tz:Alg and $\mathrm{Nb}: \mathrm{Alg}$ were dissolved separately in

MES buffer to $3 \%(\mathrm{w} / \mathrm{v})$ and MSR vaccines was resuspended to a total of $75 \mu 1$. All components

were mixed and allowed to gel for $2 \mathrm{~h}$. MSR monolith and Alginate-MSR were surgically

implanted in the subcutaneous space of mice.

Statistical analysis. All values in the present study were expressed as mean \pm S.D. Statistical analysis was performed using GraphPad Prism and Microsoft Excel. For serum antibody analysis, significance was determined using unpaired Mann-Whitney tests. For all other analyses comparing multiple groups, one-way ANOVA tests were performed. For all statistical tests, pvalues $<0.05$ considered significant.

\section{Acknowledgements.}

This work was funded by the National Institute of Health (R01 CA223255 and U54 CA244726), the Wyss Institute for Biologically Inspired Engineering, the Found Animals Foundation (D1213-

W13), and a National Science Foundation Graduate Research Fellowship to AL. The authors 
would like to thank Charles Vidoudez and the Harvard Bauer Core Facility for their help with mass spectrometry, and Dr. Thomas Conlon, Dr. Soumya Badrinath for their helpful discussions.

Conflict of Interests. Several authors are inventors on patent applications related to this technology (A.W.L, E.J.D, D.J.M); Novartis, sponsored research (D.J.M); Immulus, equity (D.J.M.) 


\section{References}

1. Wong, K. K., Li, W. W. A., Mooney, D. J. \& Dranoff, G. Advances in Therapeutic Cancer Vaccines. in Advances in Immunology (2016). doi:10.1016/bs.ai.2015.12.001

2. McHugh, K. J., Guarecuco, R., Langer, R. \& Jaklenec, A. Single-injection vaccines: Progress, challenges, and opportunities. J. Control. Release (2015). doi:10.1016/j.jconrel.2015.07.029

3. Robbins, A. \& Freeman, P. Obstacles to developing vaccines for the Third World. Sci. Am. (1988). doi:10.1038/scientificamerican1188-126

4. Kemp, J. M. et al. Continuous antigen delivery from controlled release implants induces significant and anamnestic immune responses. Vaccine (2002). doi:10.1016/S0264$410 X(01) 00444-3$

5. Baras, B., Benoit, M. A. \& Gillard, J. Parameters influencing the antigen release from spray-dried poly(DL- lactide) microparticles. Int. J. Pharm. (2000). doi:10.1016/S03785173(00)00363-X

6. Jaganathan, K. S. et al. Development of a single dose tetanus toxoid formulation based on polymeric microspheres: A comparative study of poly(D,L-lactic-co-glycolic acid) versus chitosan microspheres. Int. J. Pharm. (2005). doi:10.1016/j.ijpharm.2004.12.026

7. Wang, T. T. et al. Vaccination with a synthetic peptide from the influenza virus hemagglutinin provides protection against distinct viral subtypes. Proc. Natl. Acad. Sci. U. S. A. (2010). doi:10.1073/pnas.1013387107

8. Purcell, A. W., McCluskey, J. \& Rossjohn, J. More than one reason to rethink the use of peptides in vaccine design. Nature Reviews Drug Discovery (2007). doi:10.1038/nrd2224

9. Shaw, A. Alternative Methods of Making Influenza Vaccines. in The Bridge (The National Academies Press, 2006). 
10. Matthews, J. T. Egg-Based Production of Influenza Vaccine: 30 Years of Commercial Experience. in The Bridge (The National Academies Press, 2006).

11. Scannon, P. J. Pharmaceutical Preparedness for a Pandemic. in The Bridge (The National Academies Press, 2006).

12. Melief, C. J. M. \& Van Der Burg, S. H. Immunotherapy of established (pre)malignant disease by synthetic long peptide vaccines. Nature Reviews Cancer (2008). doi:10.1038/nrc2373

13. Kumai, T., Kobayashi, H., Harabuchi, Y. \& Celis, E. Peptide vaccines in cancer - old concept revisited. Current Opinion in Immunology (2017). doi:10.1016/j.coi.2016.11.001

14. Talwar, G. P., Vyas, H. K., Purswani, S. \& Gupta, J. C. Gonadotropin-releasing hormone/human chorionic gonadotropin $\beta$ based recombinant antibodies and vaccines. $J$. Reprod. Immunol. (2009). doi:10.1016/j.jri.2009.08.008

15. Emons, G. et al. GnRH antagonists in the treatment of gynecological and breast cancers. in Endocrine-Related Cancer (2003). doi:10.1677/erc.0.0100291

16. Aguilar, F. F. et al. Very Small Size Proteoliposomes (VSSP) and Montanide combination enhance the humoral immuno response in a GnRH based vaccine directed to prostate cancer. Vaccine (2012). doi:10.1016/j.vaccine.2012.08.020

17. Hsu, C. T. et al. Vaccination against gonadotropin-releasing hormone (GnRH) using toxin receptor-binding domain-conjugated GnRH repeats. Cancer Res. (2000).

18. Simms, M. S. et al. Anti-GnRH antibodies can induce castrate levels of testosterone in patients with advanced prostate cancer. Br. J. Cancer (2000). doi:10.1054/bjoc.2000.1315

19. Li, J. et al. Vectored antibody gene delivery mediates long-term contraception. Current Biology (2015). doi:10.1016/j.cub.2015.08.002

20. Walker, J. et al. Totally synthetic peptide-based immunocontraceptive vaccines show 
activity in dogs of different breeds. Vaccine (2007). doi:10.1016/j.vaccine.2007.07.047

21. Gupta, S. K., Shrestha, A. \& Minhas, V. Milestones in contraceptive vaccines development and hurdles in their application. Human Vaccines and Immunotherapeutics (2014). doi:10.4161/hv.27202

22. Moon, J. J. et al. Interbilayer-crosslinked multilamellar vesicles as synthetic vaccines for potent humoral and cellular immune responses. Nat. Mater. (2011). doi:10.1038/nmat2960

23. Irvine, D. J., Hanson, M. C., Rakhra, K. \& Tokatlian, T. Synthetic Nanoparticles for Vaccines and Immunotherapy. Chemical Reviews (2015). doi:10.1021/acs.chemrev.5b00109

24. Li, W. A. \& Mooney, D. J. Materials based tumor immunotherapy vaccines. Current Opinion in Immunology (2013). doi:10.1016/j.coi.2012.12.008

25. Ali, O. A., Huebsch, N., Cao, L., Dranoff, G. \& Mooney, D. J. Infection-mimicking materials to program dendritic cells in situ. Nat. Mater. (2009). doi:10.1038/nmat2357

26. Bencherif, S. A. et al. Injectable cryogel-based whole-cell cancer vaccines. Nat. Commun. (2015). doi:10.1038/ncomms8556

27. Ali, O. A., Emerich, D., Dranoff, G. \& Mooney, D. J. In situ regulation of DC subsets and T cells mediates tumor regression in mice. Sci. Transl. Med. (2009). doi:10.1126/scitranslmed.3000359

28. Kim, J. et al. Injectable, spontaneously assembling, inorganic scaffolds modulate immune cells in vivo and increase vaccine efficacy. Nat. Biotechnol. (2015). doi:10.1038/nbt.3071

29. Li, A. W. et al. A facile approach to enhance antigen response for personalized cancer vaccination. Nat. Mater. (2018). doi:10.1038/s41563-018-0028-2

30. Swaminathan, A., Lucas, R. M., Dear, K. \& McMichael, A. J. Keyhole limpet haemocyanin - A model antigen for human immunotoxicological studies. Br. J. Clin. 
Pharmacol. (2014). doi:10.1111/bcp.12422

31. Kurosaki, T., Kometani, K. \& Ise, W. Memory B cells. Nat. Rev. Immunol. (2015). doi:10.1038/nri3802

32. Pape, K. A., Taylor, J. J., Maul, R. W., Gearhart, P. J. \& Jenkins, M. K. Different B cell populations mediate early and late memory during an endogenous immune response.

Science (80-. ). (2011). doi:10.1126/science. 1201730

33. Hartmann-Boyce, J., Cahill, K., Hatsukami, D. \& Cornuz, J. Nicotine vaccines for smoking cessation. Cochrane Database of Systematic Reviews (2012). doi:10.1002/14651858.CD007072.pub2

34. Hwang, C. S. et al. Enhancing Efficacy and Stability of an Antiheroin Vaccine: Examination of Antinociception, Opioid Binding Profile, and Lethality. Mol. Pharm. (2018). doi:10.1021/acs.molpharmaceut.7b00933

35. Desai, R. I. \& Bergman, J. Nicotine-Targeting Nano-Vaccines for Smoking Cessation. Neuropsychopharmacology (2016). doi:10.1038/npp.2015.234

36. Silva, M. et al. Targeted Elimination of Immunodominant B Cells Drives the Germinal Center Reaction toward Subdominant Epitopes. Cell Rep. (2017). doi:10.1016/j.celrep.2017.12.014

37. Loi, S. et al. Effects of Estrogen Receptor and Human Epidermal Growth Factor Receptor-2 Levels on the Efficacy of Trastuzumab A Secondary Analysis of the HERA Trial. JAMA Oncol. (2016). doi:10.1001/jamaoncol.2016.0339

38. Yip, Y. L., Smith, G. \& Ward, R. L. Comparison of phage pIII, pVIII and GST as carrier proteins for peptide immunisation in Balb/c mice. Immunol. Lett. (2001). doi:10.1016/S0165-2478(01)00281-4

39. Granoff, D. M., Rathore, M. H., Holmes, S. J., Granoff, P. D. \& Lucas, A. H. Effect of 
immunity to the carrier protein on antibody responses to Haemophilus influenzae type b conjugate vaccines. Vaccine (1993). doi:10.1016/0264-410X(93)90160-Y

40. Romond, E. H. et al. Trastuzumab plus adjuvant chemotherapy for operable HER2positive breast cancer. N. Engl. J. Med. (2005). doi:10.1056/NEJMoa052122

41. Schneider, J. W., Chang, A. Y. \& Garratt, A. Trastuzumab cardiotoxicity: Speculations regarding pathophysiology and targets for further study. in Seminars in Oncology (2002). doi:10.1053/sonc.2002.34051

42. Suter, T. M. et al. Trastuzumab-associated cardiac adverse effects in the herceptin adjuvant trial. J. Clin. Oncol. (2007). doi:10.1200/JCO.2006.09.1611

43. Cho, H. S. et al. Structure of the extracellular region of HER2 alone and in complex with the Herceptin Fab. Nature (2003). doi:10.1038/nature01392

44. Dakappagari, N. K., Douglas, D. B., Triozzi, P. L., Stevens, V. C. \& Kaumaya, P. T. P. Prevention of mammary tumors with a chimeric HER-2 B-cell epitope peptide vaccine. Cancer Res. (2000).

45. Dakappagari, N. K. et al. Conformational HER-2/neu B-cell epitope peptide vaccine designed to incorporate two native disulfide bonds enhances tumor cell binding and antitumor activities. J. Biol. Chem. (2005). doi:10.1074/jbc.M411020200

46. Garrett, J. T. et al. Novel Engineered Trastuzumab Conformational Epitopes Demonstrate In Vitro and In Vivo Antitumor Properties against HER-2/ neu . J. Immunol. (2007). doi:10.4049/jimmunol.178.11.7120

47. Ali, O. A., Doherty, E., Mooney, D. J. \& Emerich, D. Relationship of vaccine efficacy to the kinetics of DC and T-cell responses induced by PLG-based cancer vaccines. Biomatter (2011). doi:10.4161/biom.1.1.16277

48. Moon, J. J. et al. Enhancing humoral responses to a malaria antigen with nanoparticle 
vaccines that expand $\mathrm{T}$ fh cells and promote germinal center induction. Proc. Natl. Acad.

Sci. U. S. A. (2012). doi:10.1073/pnas.1112648109

49. Kasturi, S. P. et al. Programming the magnitude and persistence of antibody responses with innate immunity. Nature (2011). doi:10.1038/nature09737

50. Tam, H. H. et al. Sustained antigen availability during germinal center initiation enhances antibody responses to vaccination. Proc. Natl. Acad. Sci. U. S. A. (2016). doi:10.1073/pnas.1606050113

51. Johansen, P. et al. Antigen kinetics determines immune reactivity. Proc. Natl. Acad. Sci. U. S. A. (2008). doi:10.1073/pnas.0706296105

52. Li, A. W. et al. A facile approach to enhance antigen response for personalized cancer vaccination. Nat. Mater. (2018). doi:10.1038/s41563-018-0028-2 


\section{Figures, Schemes and Legends}

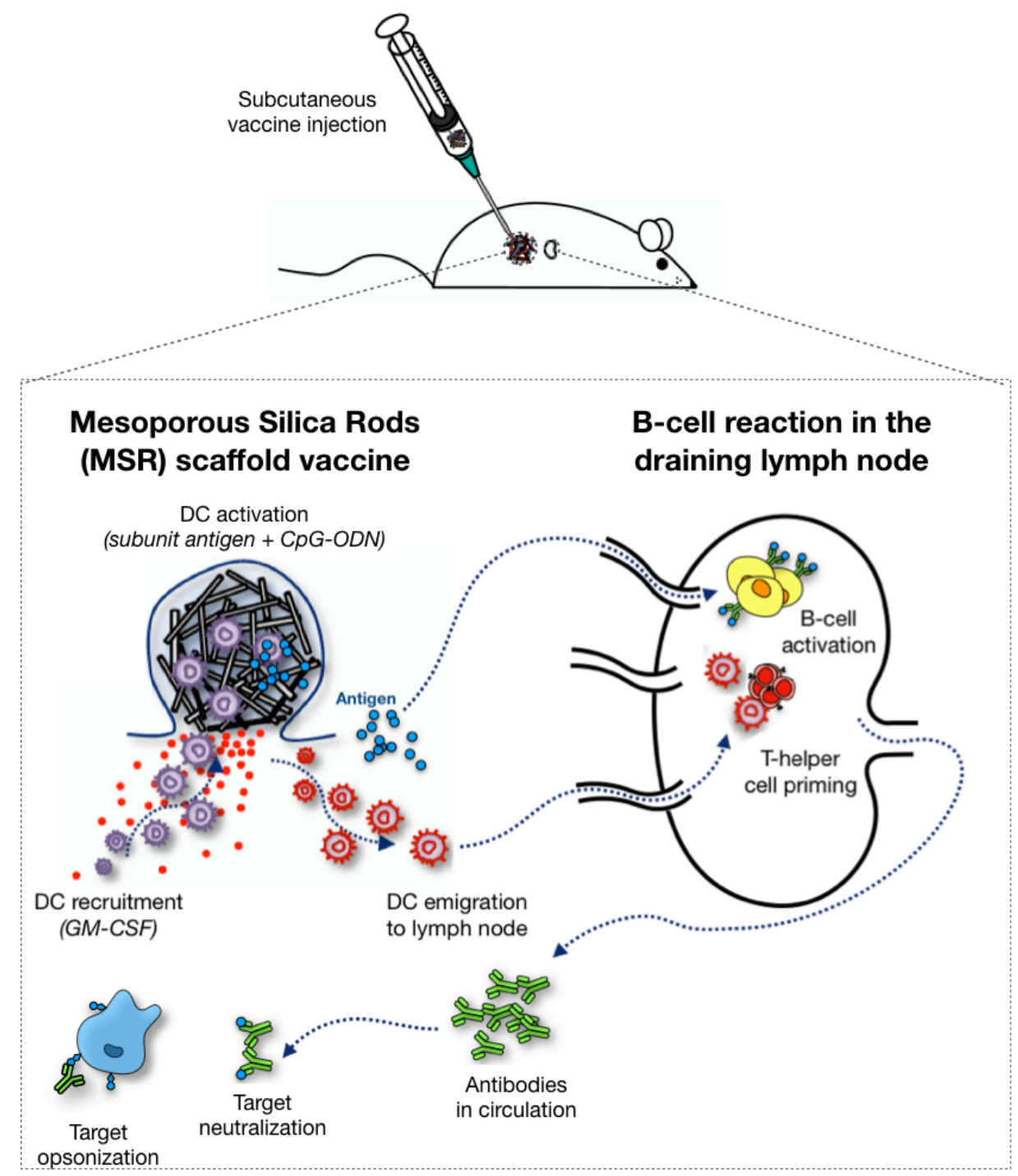

Figure 1. Mesoporous Silica Rods vaccines for humoral response generation

Mesoporous Silica Rods loaded with GM-CSF, CpG-ODN and subunit antigens are injected subcutaneously to form a 3D immunomodulating scaffold to produce antibodies against small antigen targets. MSR scaffold serves two purposes (1) as a long-term depot for sustained antigen release and presentation to B-cells in the draining lymph node. (2) as a local niche for dendritic cell (DC) recruitment where they pick up antigen and get activated by TLR-9 agonist CpG. Mature DCs can migrate to the lymph node where they prime antigen-specific T-cells that support the development of the humoral response. 
a

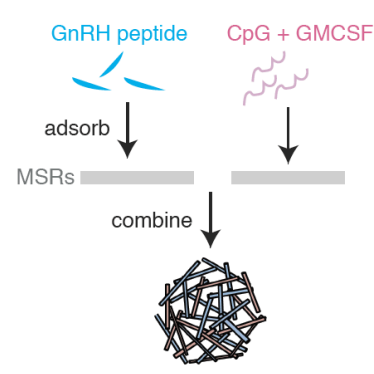

GnRH-OVA MSR Vax GnRH-OVA conjugate CpG + GMCSF

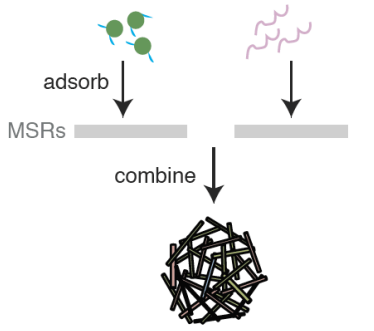

d Bolus Vax

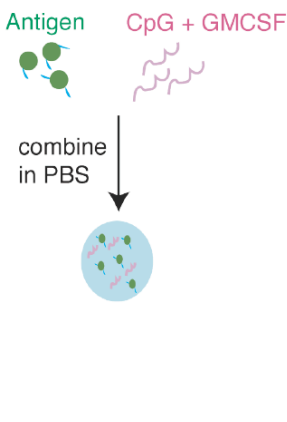

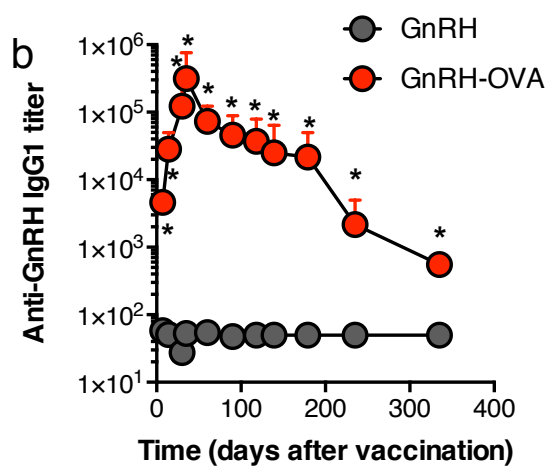

C

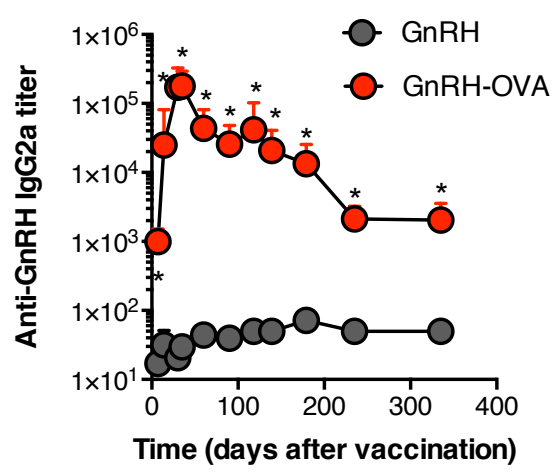

e
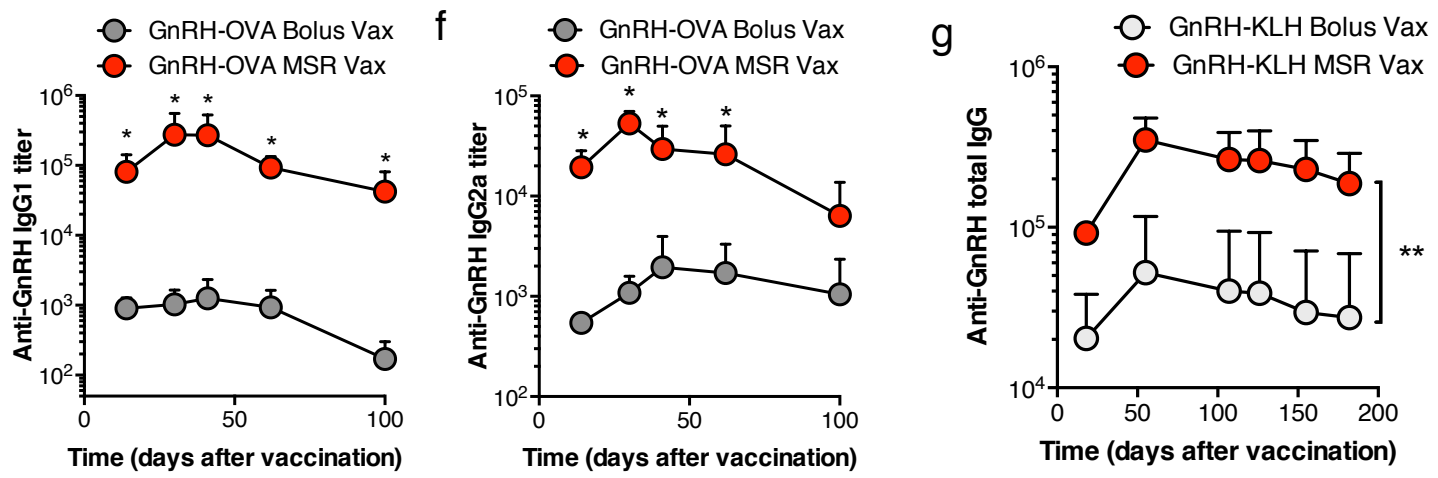

Figure 2: Single injection of MSR vaccine with GnRH-OVA induces durable anti-GnRH peptide serum antibody response, and higher titer than bolus formulations. (a) Schematic of MSR vaccines formulations using free GnRH peptide (left) or GnRH-OVA (right). (B-C) ELISA analysis of sera GnRH-specific IgG1 (b) or IgG2a (c) after immunization with MSR vaccines containing free GnRH or GnRH-OVA (mean and s.d., $n=4$ ) * indicates $p<0.05$. (d) Schematic of Bolus vaccine (left) and the MSR vaccine (right). (E-F) ELISA analysis of sera GnRHspecific IgG1 (e) or IgG2a (f) after immunization with MSR vaccines loaded with GnRH-OVA (GnRH-OVA MSR Vax) or bolus vaccine (GnRH-OVA Bolus Vax). (mean and s.d., $\mathrm{n}=4$ ) * indicates $\mathrm{p}<0.05$. (g) ELISA analysis of sera GnRH-specific total $\mathrm{IgG}$ after immunization with MSR vaccine loaded with GnRH-KLH (MSR Vax) or bolus vaccine (Bolus vax) ). mean and s.d., $\mathrm{n}=4 * *$ indicates $\mathrm{p}<0.01$ 


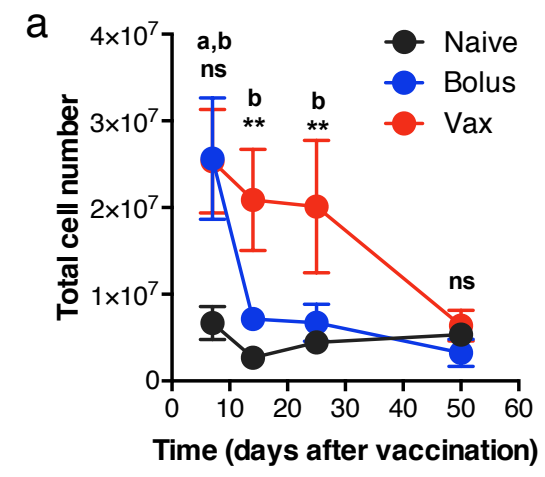

$\mathrm{d}$

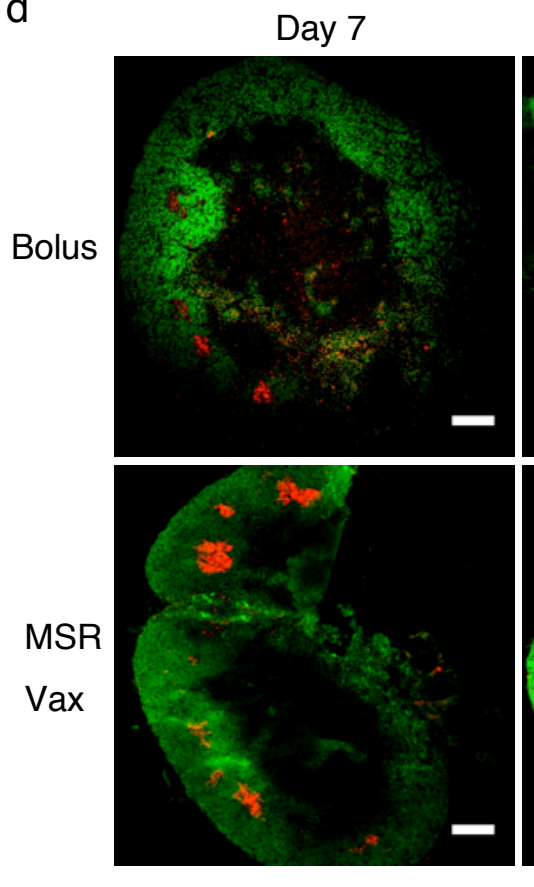

b

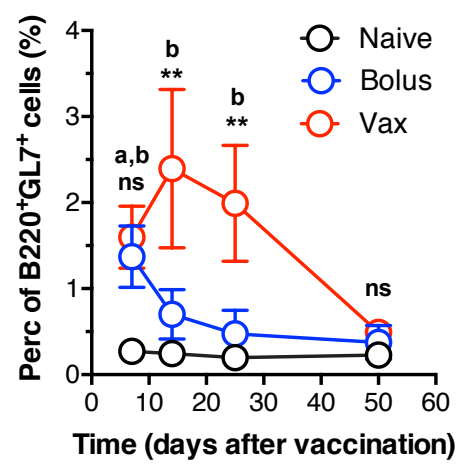

C

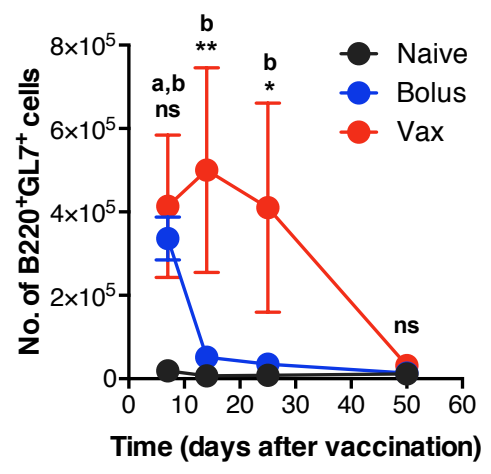

Day 14
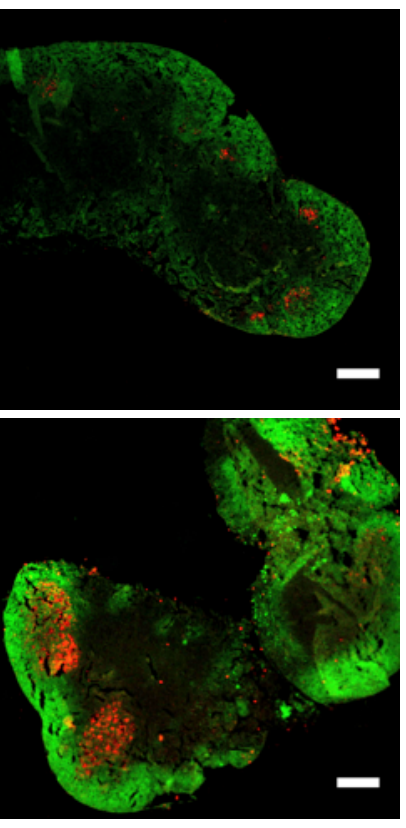

Day 25

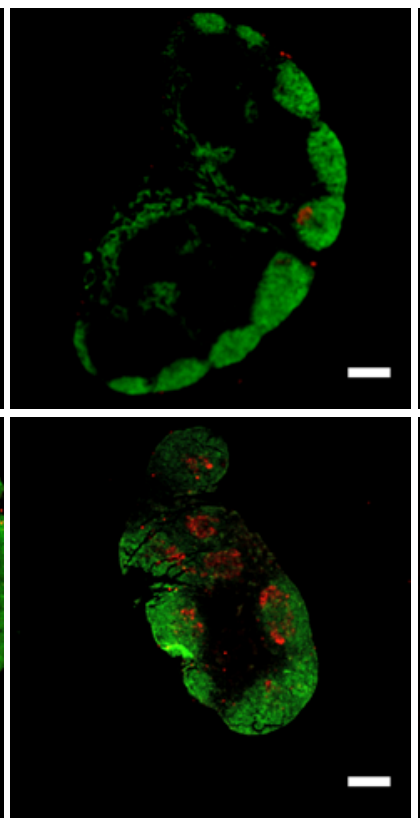

Day 50

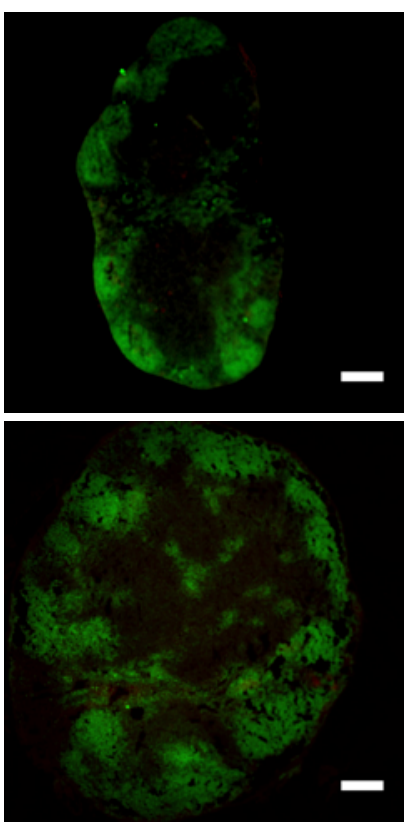

$-\mathrm{B} 220-\mathrm{GL} 7$

Figure 3: MSR vaccine with GnRH-OVA induces persistent germinal center formation. (a)

Total number of cells in the dLN of untreated mice (Naïve) or after immunizing with the MSR vaccine (vax) and bolus formulations of the vaccine (Bolus). (b) Percentage and (c) number of $\mathrm{B} 220^{+} \mathrm{GL}^{+} \mathrm{GC}$ B cells in the dLN (c) Histological staining of dLN sections stained with antiB220 and anti-GL7. scale bar $=300 \mu \mathrm{m}$. . (mean and s.d., $\mathrm{n}=4$ ) a indicates $\mathrm{p}<0.05$ between Naïve and Bolus group, $b$ indicates $p<0.05$ between Naïve and Vax, * indicates $p<0.05, * *$ $\mathrm{p}<0.01$ and ns, no significance between Bolus and Vax 

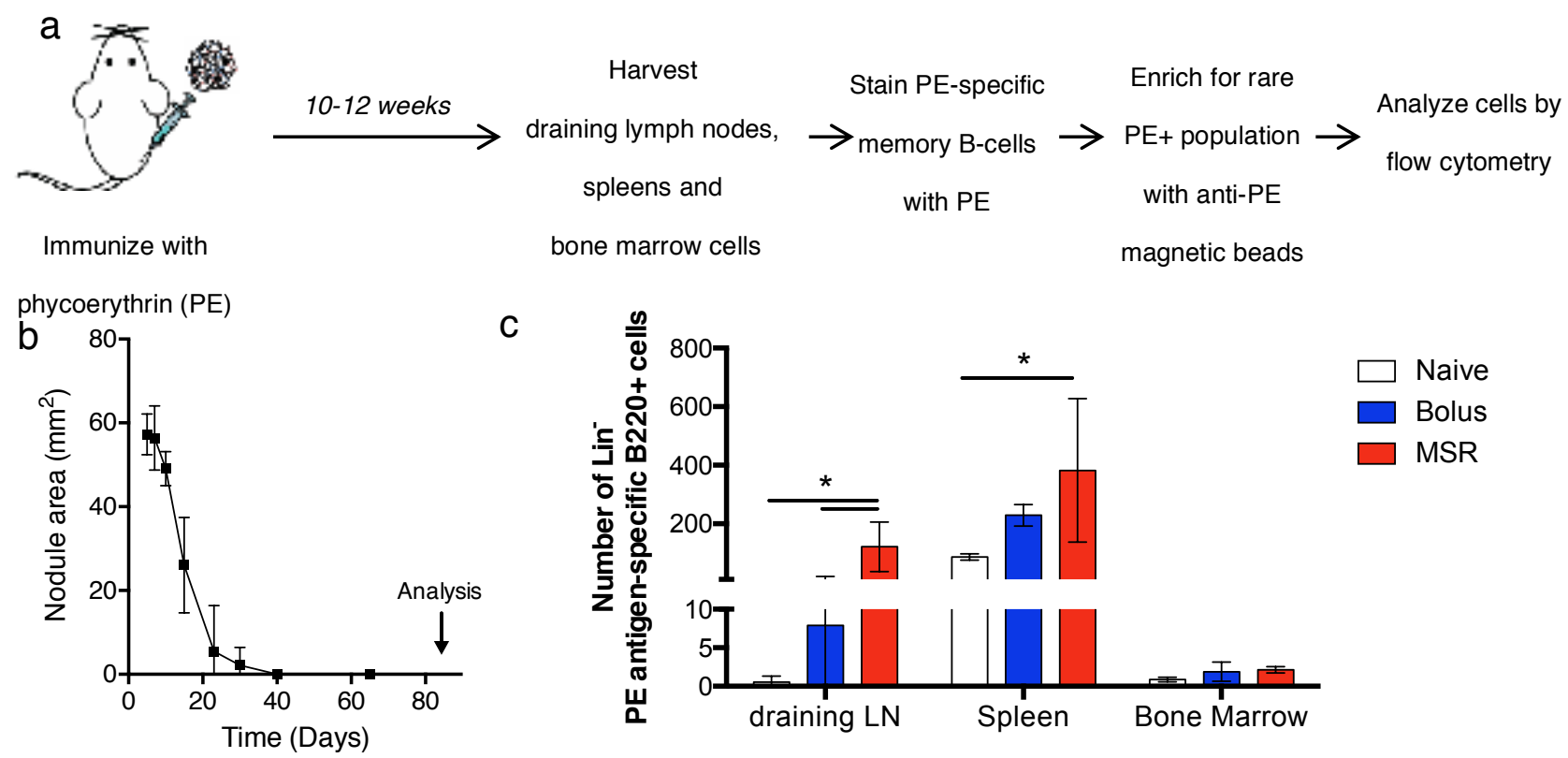

C

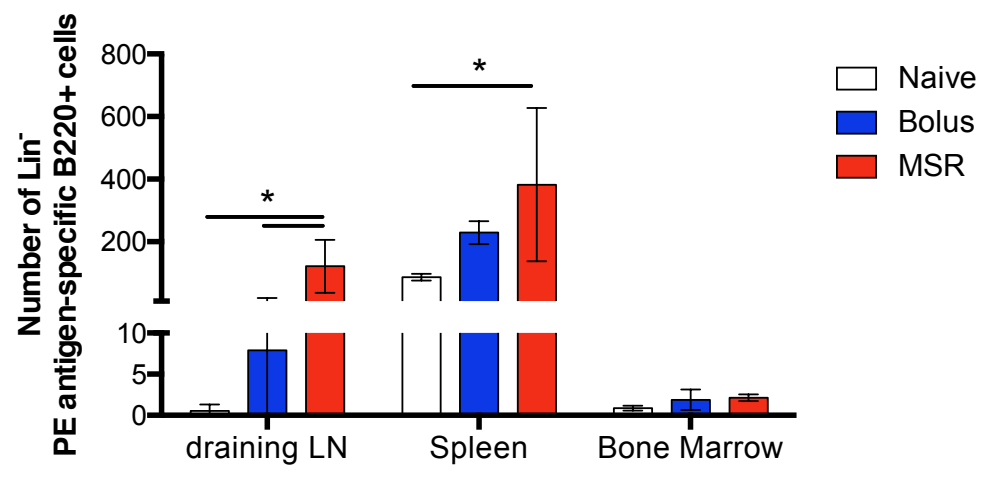

Figure 4: MSR vaccine improves memory B-cell generation (a) Scheme of experimental design.

Mice were left untreated (naïve) or received a phycoerythrin (PE)-containing vaccine in soluble (Bolus) or scaffold form (MSR). 10-12 weeks after immunization, when the vaccine components have cleared, cells of the draining lymph nodes, spleens and bone marrow were stained with PE to tag PE-binding cells. Anti-PE magnetic beads were used to enrich for the are PE-specific memory B-cells and further stained for analysis by flow cytometry. (b) Scaffold size over time, data represents mean and $\mathrm{SD}, \mathrm{n}=4$. (c) Total number of PE-specific Lin-B220 ${ }^{+}$cells (Lin- represents CD4-CD8-F4/80-Gr1-Cd11c- live cells) in the draining lymph nodes, spleens and bone marrow of naïve and bolus or MSR vaccinated mice, 83 days after immunization. (data represents individual data point with mean), $\mathrm{n}=4$. * indicates $\mathrm{p}<0.05$. 
a

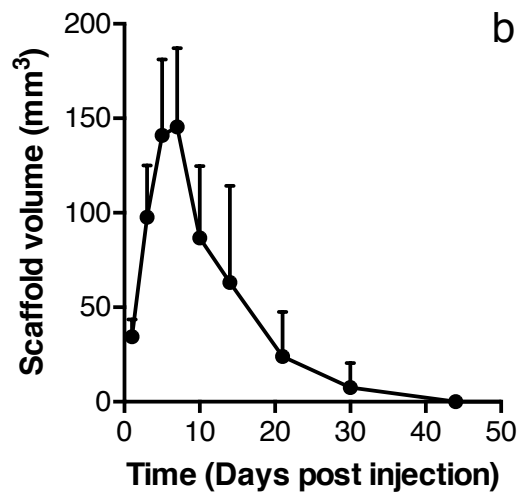

b

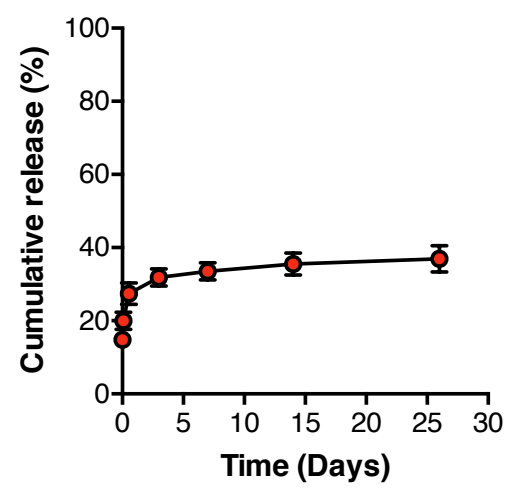

C

Day 7

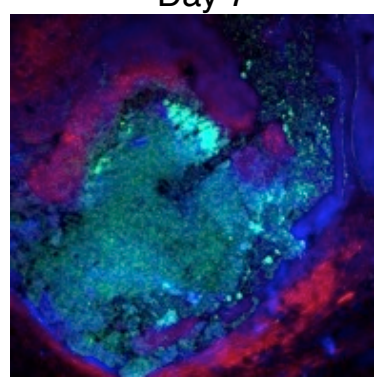

d

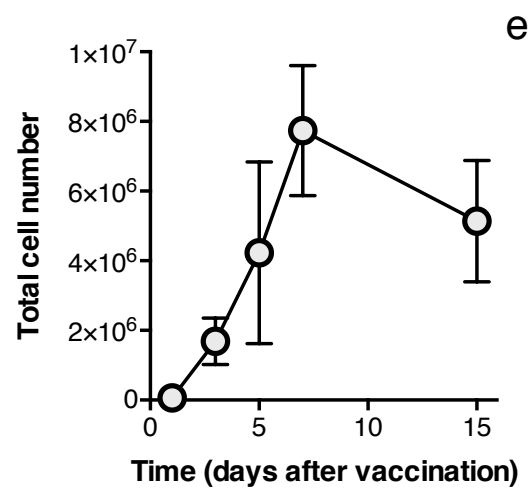

e
Day 15
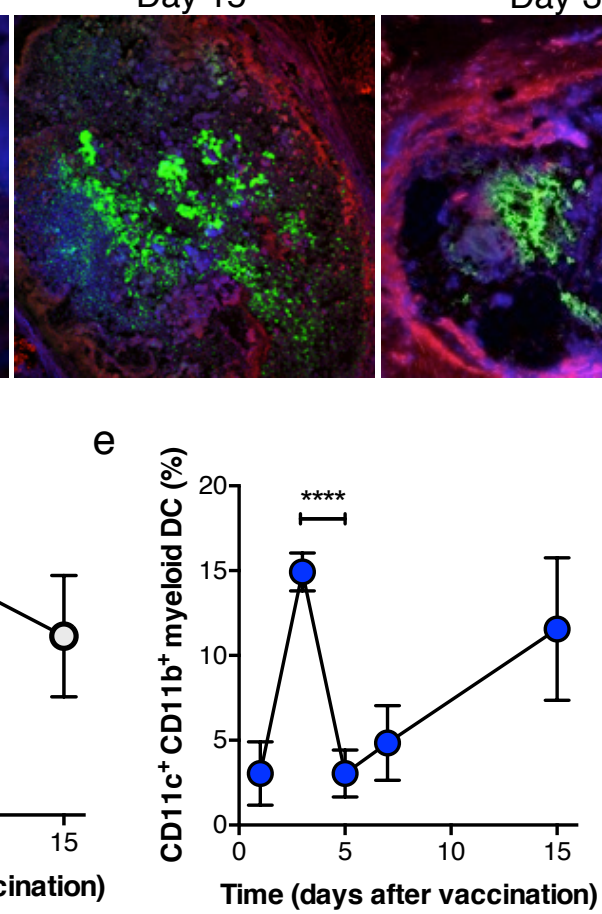

Day 30

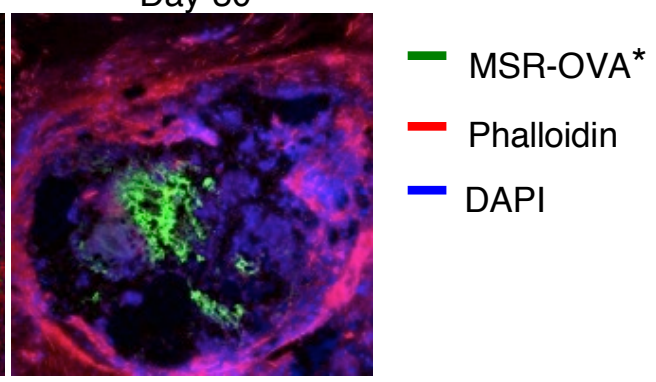

$f$

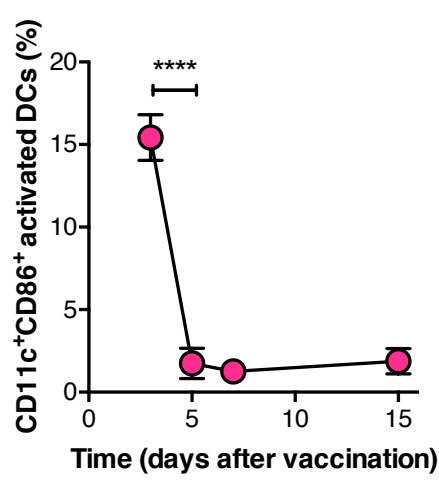

Figure 5: MSRs form dynamic scaffolds for sustained antigen release and DC recruitment and activation. (a) MSR vaccine scaffold volume over time. (mean and SD, $n=4$ ), (b) In vitro cumulative release of GnRH-OVA antigen from MSR scaffold. (mean and SD, n=3) (c) Representative confocal images of explanted MSR vaccine scaffolds containing fluorescently

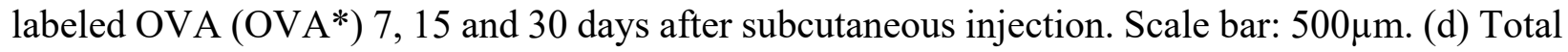
number of cells in the MSR vaccine scaffold over time. (e) Percentage of CD11 $\mathrm{c}^{+} \mathrm{CD} 11 \mathrm{~b}^{+}$myeloid DCs in the MSR vaccine scaffold over time. (f) Percentage of CD11 $\mathrm{c}^{+} \mathrm{CD} 86^{+}$activated DCs in the MSR vaccine scaffold over time (mean and SD, $n=4$ ), **** indicates $\mathrm{p}<0.0001$ 

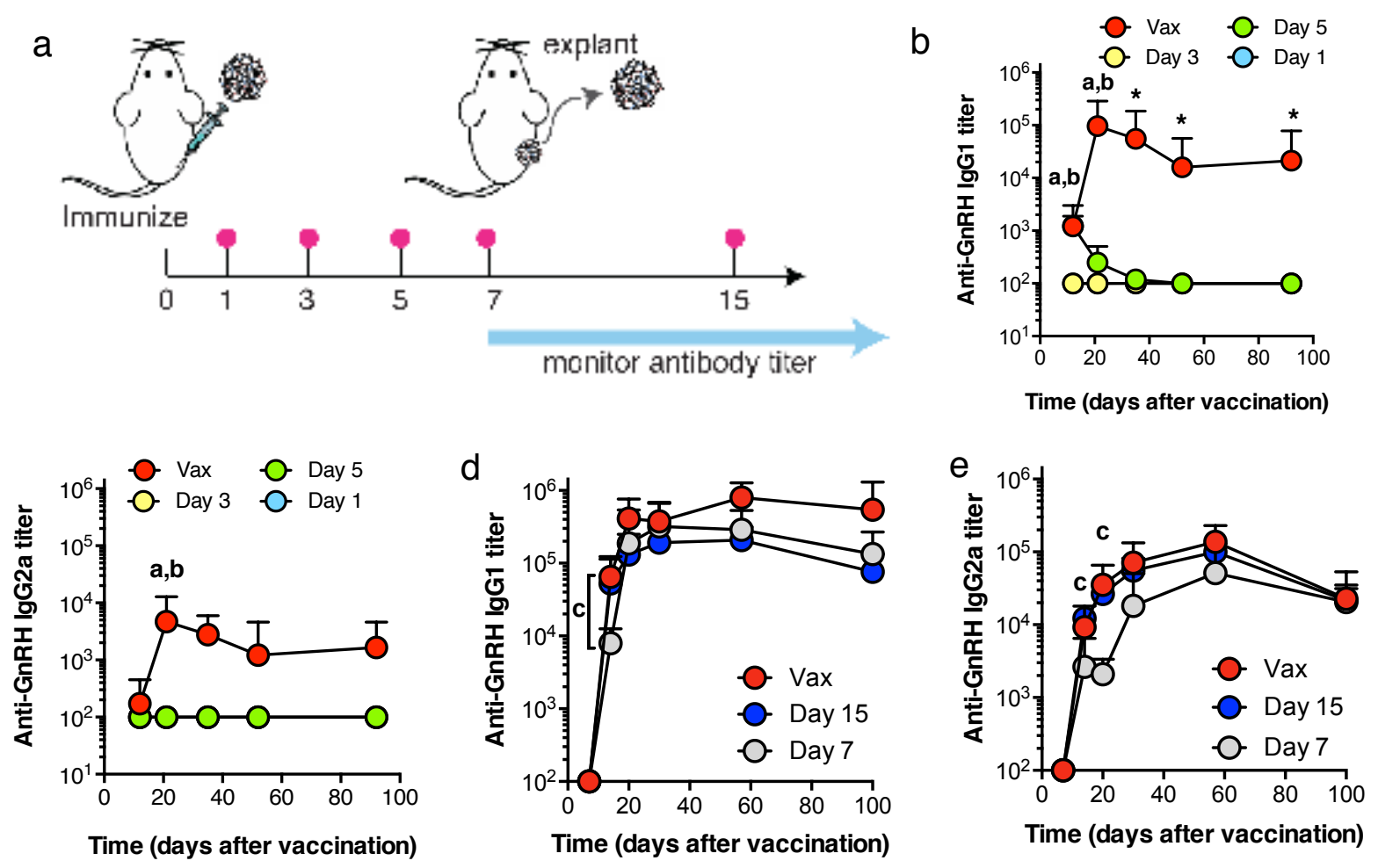

Figure 6: MSR vaccine duration impacts anti-GnRH antibody production. (a) Schematics of MSR vaccine regimen. Vaccine duration is varied from 1, 3, 5, 7 and 15 days, or indefinitely. (bc) ELISA analysis of sera GnRH-specific IgG1 (b) or IgG2a (c) antibody from mice immunized with the MSR vaccine for a duration of 1 (Day 1), 3 (Day 3), 5 days (Day 5) or indefinitely (Vax). (d-e). ELISA analysis of sera GnRH-specific IgG1 (d) or IgG2a (e) antibody from mice immunized with the MSR vaccine for a duration of 7 days (Day 7), 15 days (Day 15) or indefinitely (Vax). (mean and s.d., $\mathrm{n}=4$ ) a indicates $\mathrm{p}<0.05$ between Vax and Day 1, b between Vax and Day 3, c between Vax and Day 7 and * between Vax and all other groups. 


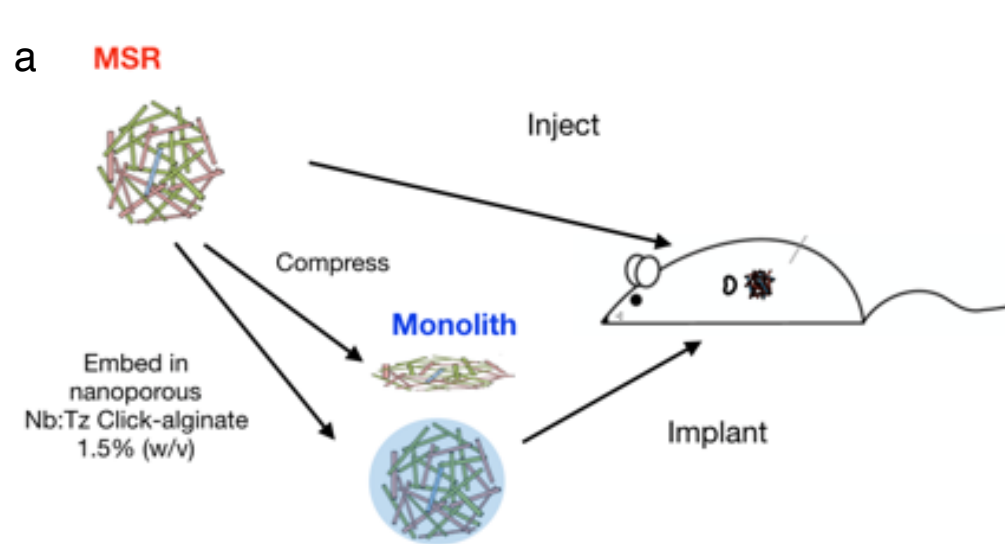

Alginate-MSR b

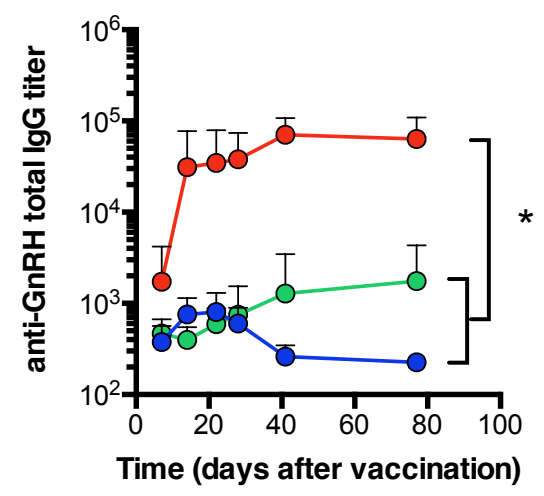

MSR

Alginate-MSR

Figure 7: Reduced cell infiltration impairs antibody response. (a) Schematics of the modification of MSR vaccine to reduce cell infiltration. Lyophilized MSR vaccines were individually compressed into a 8mm-diameter disk using a lab press (monolith) or encapsulated into a nanoporous alginate hydrogel and implanted surgically in the subcutaneous space on mice's flank (b) ELISA analysis of sera anti-GnRH total IgG titer over time. MSR condition indicates control MSR vaccine (not compressed nor incorporated into gel). All vaccine conditions contained the same masses of GM-CSF and antigen. (mean and SD, n=4), ${ }^{*}, \mathrm{p}<0.05$ for all timepoints $>7$ days. 


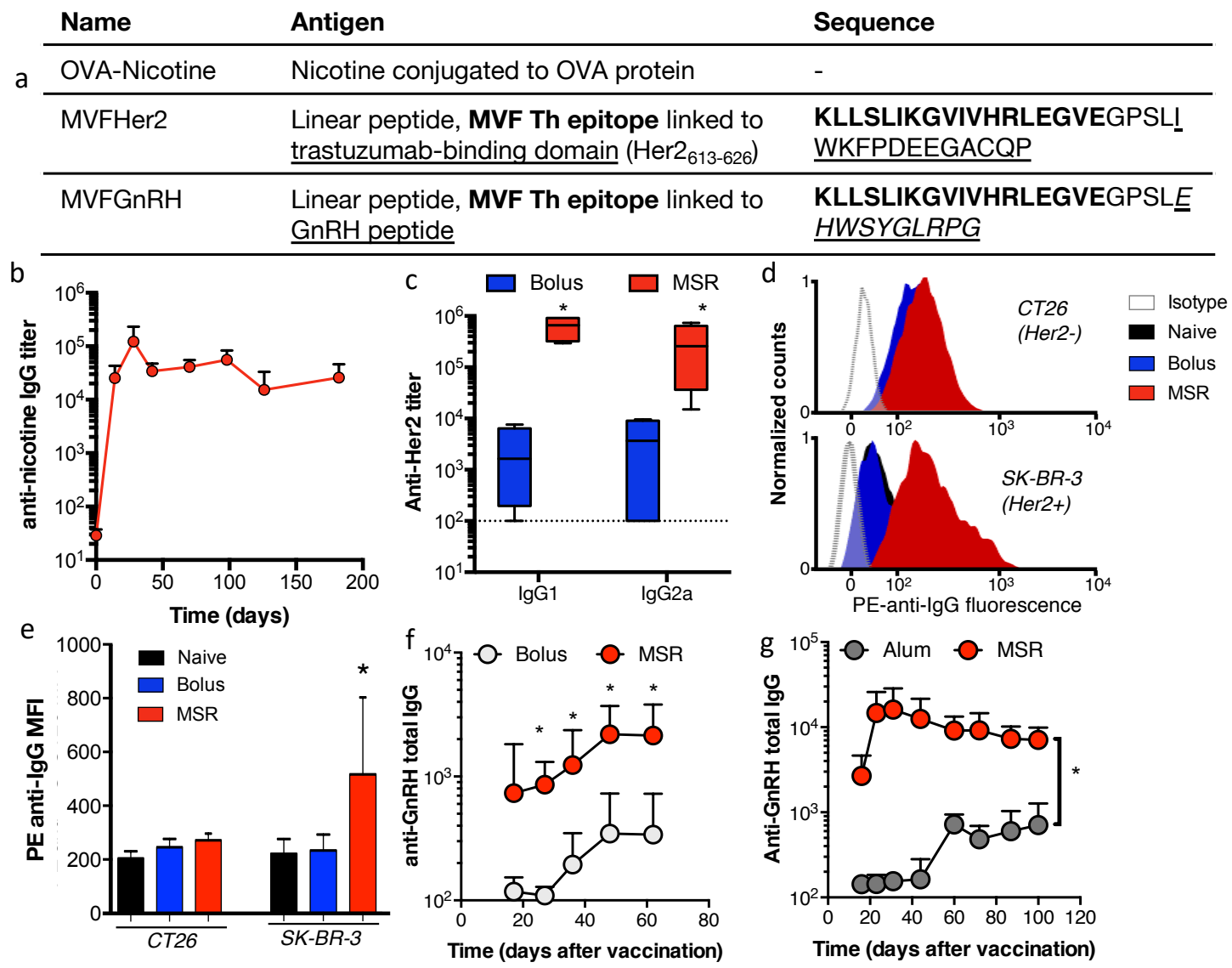

Figure 8: MSR scaffold vaccines induce robust humoral responses against a wide variety of small antigenic therapeutic targets (a) Details of small molecule or linear peptide antigens used in vaccination studies. (b). ELISA analysis of anti-nicotine total IgG titer over time following vaccination using MSR vaccine. (c) ELISA analysis of anti-Her2 antibody on day 23 after vaccination with bolus (Bolus, $n=4$ ) or MSR vaccine (MSR, $n=5$ ); containing MVFHer2. Dotted line represents antibody levels in naïve mice; data represents mean and $\mathrm{SD}$, * indicates $\mathrm{p}<0.05$. (df) Flow cytometry analysis of serum IgG binding to non-Her2 expressing CT26 cells or Her2 overexpressing SK-BR-3 cells. (d) Representative anti-IgG fluorescence after treatment with serum from untreated (naïve) mice or animals immunized with a bolus (Bolus) or MSR (MSR) vaccine containing MVFHer2. (e) Quantification of IgG binding to CT26 and SK-BR-3 cells incubated with the serum of naïve (Naïve, $n=5$ ) or MVFHer2 vaccinated mice (Bolus, $n=4$ or MSR, $\mathrm{n}=5$ ) * indicates $\mathrm{p}<0.05$. (f-g) ELISA analysis of serum anti-GnRH IgG over time comparing mice immunized with a bolus (bolus, $n=4$ ) or MSR vaccine (MSR, $n=4)$ containing MVFGnRH (f) or (g) MVFGnRH formulated in standard Alum adjuvant (Alum, $n=4$ ) and the MSR vaccine (MSR, $\mathrm{n}=4$ ). data represents mean and $\mathrm{SD}$; indicates $\mathrm{p}<0.05$ 